\title{
Limits and options for electronic devices for the treatment of hydrocephalus Christoph Miethke
}

Address: Christoph Miethke GmbH\&Co. KG, Ulanenweg 2, D-144690 Potsdam, Germany

Email: Christoph Miethke - christoph.miethke@miethke.com

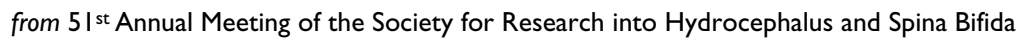
Heidelberg, Germany. 27-30 June 2007

Published: 20 December 2007

Cerebrospinal Fluid Research 2007, 4(SuppI I):S3I doi:I0.II86/I743-8454-4-SI-S3 I

This abstract is available from: http://www.cerebrospinalfluidresearch.com/content/4/SI/S3I

(c) 2007 Miethke; licensee BioMed Central Ltd.

\section{Background}

The available shunt-systems for the treatment of hydrocephalus are exclusively based on simple mechanical principles. In comparison to pacemakers or other sophisticated medical devices, shunt-technology is said to be "old-fashioned" and the introduction of electronic intelligent systems should be a second remarkable breakthrough after the introduction of the first shunts in the beginning of the fifties. The decisive question is how far an electronically controlled device can present new possibilities for the treatment without a significant increase of new risks. Which concept promises significant improvements?

\section{Materials and methods}

All available shunt-systems operate depending on the pressure difference between the ventricular system and the abdominal cavity or the atrium of the heart. The used mechanical principles react to changes of this differential pressure. The goal is to re-establish physiological pressure values in the ventricular system of the patient. The obvious solution to control the intra-ventricular pressure (IVP) electronically is the opening and closing of the device depending on the actual measured IVP. But this simple approach leads to severe unsolved technical problems like long-term drift and general accuracy. It is a matter of controversy whether a shunt-system should reestablish a certain IVP, a certain ventricular size or possibly a certain condition of the brain tissue. However, the main purpose of drainage is the withdrawal of CSF from the ventricular system. The amount might depend on changing individual conditions or on age, on the height of the patient or on the kind of hydrocephalus. The introduction of an electronically controlled programmable switch presents new perspectives for the diagnosis, the general understanding and the therapy of hydrocephalus. The switch works without a pressure transducer. The device is programmed by a physician, who determines at what time during the day or the night the shunt without a valve is open or closed. The shunt can be closed at any time noninvasively or it can be programmed to be always open. The telemetric programming allows any kind of individual adaptation. Because a battery is needed, the size of the device has to be similar to pacemakers. Therefore, it is recommended to implant the switch in the chest of the patient.

\section{Results and conclusion}

The concept of an electronically controlled switch broadens the possibilities of the treatment of hydrocephalus. This clear advantage might be counterbalanced by the drawbacks like the big housing needed or the new risks introduced by the electronic components. The first implantations of the new device are scheduled to be performed in the beginning of 2006. If the clinical results confirm the benefits of the concept the electronic switch will be an option especially for adult patients with NPH. 